\title{
Utilização de Chlorimuron-Ethyl E Imazethapyr Na CUltura da SOJA ROUNDUP READY ${ }^{\circledR 1}$
}

\author{
Chlorimuron-ethyl and Imazethapyr Applied on Roundup Ready ${ }^{\circledR}$ Soybean Crop
}

PROCÓPIO, S.O. ${ }^{2}$, MENEZES, C.C.E. ${ }^{3}$, BETTA, L. ${ }^{4}$ e BETTA, M. ${ }^{4}$

\begin{abstract}
RESUMO - Objetivou-se com este trabalho avaliar a eficácia e a seletividade da associação dos herbicidas chlorimuron-ethyl e imazethapyr ao glyphosate no controle de plantas daninhas na cultura da soja Roundup Ready ${ }^{\circledR}\left(R^{\circledR}\right)$. O experimento foi realizado no Centro Tecnológico da COMIGO, localizado no municipio de Rio Verde-GO, na safra 2005/2006. A semeadura do cultivar Monsoy 7878 foi realizada mecanicamente, sendo a semeadora regulada para liberar 18 sementes por metro. O espaçamento utilizado entre linhas foi de $0,50 \mathrm{~m}$. O delineamento experimental foi o de blocos ao acaso, com quatro repetições. A aplicação isolada de glyphosate nas doses de 480, 960 e $1.440 \mathrm{~g}^{\text {ha }}{ }^{-1}$ não causou intoxicação e não reduziu a altura e o acúmulo de massa seca das plantas de soja RR $^{\circledR}$. A adição dos herbicidas imazethapyr e chlorimuron-ethyl ao glyphosate ocasionou intoxicação às plantas de soja $R^{\circledR}$, todavia em níveis aceitáveis, sendo os sintomas provocados pela ação do imazethapyr mais intensos em comparação aos do chlorimuron-ethyl. A associação do herbicida imazethapyr ao glyphosate reduziu a altura e o acúmulo de fitomassa das plantas de soja $R^{\circledR}$. A aplicação isolada de glyphosate nas doses de 480, 960 e $1.440 \mathrm{~g} \mathrm{ha}^{-1}$ não apresentou controle satisfatório das plantas daninhas Euphorbia heterophylla, Commelina benghalensis, Chamaesyce hirta, Leucas martinicensis e Ipomoea grandifolia. Apesar de incrementar o controle da maioria das plantas daninhas, a adição dos herbicidas chlorimuronethyl e imazethapyr ao glyphosate não promoveu aumento de produtividade de grãos na cultura da soja $R R^{\circledast}$.
\end{abstract}

Palavras-chave: glyphosate, Glycine max, plantas daninhas, fitotoxicidade.

ABSTRACT - The objective of this work was to evaluate the efficacy and selectivity of the herbicides chlorimuron-ethyl and imazethapyr associated to glyphosate in controlling weeds on Roundup Ready $\left(R R^{\circledR}\right)$ soybean crop. The experiment was carried out at the COMIGO Technological Center in Rio Verde-GO, during the 2005/2006-crop season. The variety used was Monsoy 7878 which had 18 seeds mechanically distributed per meter. The rows were spaced by $0.50 \mathrm{~m}$. The experiment was arranged in a completely randomized block design with four replications for each treatment. Glyphosate doses (480, 960 and $1.440 \mathrm{~g} \mathrm{ha}^{-1}$ ), applied alone, did not cause plant toxicity nor reduced height and biomass accumulation of $R R^{\circledR}$ soybean plants. However, the addition of the herbicides imazethapyrand chlorimuronethyl to glyphosate caused plant toxicity in the soybean $R R$ plants, though at an acceptable degree. The symptoms caused by imazethapyr were more severe than those caused by chlorimuron-ethyl. The association of imazethapyr and glyphosate led to reduced heightand biomass. The application of glyphosate alone at doses 480,960 and $1.440 \mathrm{~g} \mathrm{ha}$ ${ }^{1}$, did not exert sufficient control over Euphorbia heterophylla, Commelina benghalensis, Chamaesyce hirta, Leucas martinicensis and Ipomoea grandifolia. Despite increasing control of most of the weeds, chlorimuron-ethyl and imazethapyr addition did not promote increased yield when applied on the $R R^{\circledR}$ soybean crop.

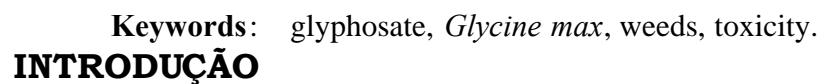

Recebido para publicação em 19.9.2006 e na forma revisada em 17.4.2007.

2 Pesquisador da Embrapa Tabuleiros Costeiros. 49.025-040, Aracaju-SE. <procopio@ @patc.embrapa.br>; ${ }^{3}$ Eng $^{2}$-Agr ${ }^{\mathrm{e}}$ da Cooperativa Agroindustrial dos Produtores Rurais do Sudoeste Goiano. <cmenezes@ fesurv.br>; ${ }^{4}$ Discente do curso de Agronomia da Fesurv - Universidade de Rio Verde.

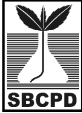

Planta Daninha, Viçosa-MG v. 25, n. 2, p. 365-373, 2007 
A introdução no mercado brasileiro das variedades de soja transgênicas - mais especificamente aquelas que possuem o gene de tolerância ao herbicida glyphosate - gerou profundas modificações no controle químico de plantas daninhas (Gazziero et al., 2006). Devido às características positivas do herbicida glyphosate, como ação em pós-emergência tanto inicial como tardia, alta sistematicidade e amplo espectro de ação, técnicos e agricultores acreditaram que os problemas no manejo de plantas daninhas na cultura da soja estariam resolvidos com a introdução das variedades Roundup Ready ${ }^{\circledR}\left(R^{\circledR}\right)$. No entanto, o controle insatisfatório de algumas espécies de plantas daninhas em aplicações de dessecação de manejo, no sistema de plantio direto com esse herbicida, já mostrava indícios de que, mesmo sendo um excelente produto, associações com outros princípios ativos poderiam ser benéficas. Espécies invasoras, como ervaquente (Spermacoce latifolia), agriãozinho (Synedrellops is grisebachii), trapoe raba (Commelina benghalensis) e erva-de-touro (Tridax procumbens), vêm sendo selecionadas devido a aplicações sucessivas de glyphosate nas áreas agrícolas do cerrado. De acordo com Johnson et al. (2002), as falhas no controle de determinadas plantas daninhas pelo uso do glyphosate têm levado agricultores a aplicar outros herbicidas, mesmo em lavouras de soja $R^{\circledR}$.

Entre as alternativas para incrementar a eficiência no controle de espécies que apresentam certa tolerância ao glyphosate, podem ser utilizados os herbicidas chlorimuron-ethyl e imazethapyr, que já vêm sendo associados ao glyphosate em aplicações de dessecação de manejo (Vanlieshout \& Loux, 2000).

Os herbicidas chlorimuron-ethyl e imazethapyr, apesar de pertencerem a grupos químicos diferentes, possuem o mesmo mecanismo de ação, atuando na inibição da enzima acetolactato sintase (ALS), que atua na rota de sintese dos aminoácidos ramificados valina, leucina e isoleucina. Apres entam controle, principalmente, de espécies daninhas latifoliadas, normalmente mais tolerantes ao glyphosate. Trabalhos conduzidos por Norsworthy \& Grey (2004) mostram que a adição de chlorimuron-ethyl ao glyphosate proporcionou incremento no controle de
Ipomoea hederacea e Ipomoea purpurea. Vidrine et al. (2002) verificaram que a associação de chlorimuron-ethyl ao glyphosate incrementou o controle de Ipomoea hederacea em 5\%, enquanto no controle de Sesbania exaltata o aumento foi de 12\%. Vangessel et al. (2001) relatam que, em relação à aplicação isolada de glyphosate, a mistura em tanque de glyphosate, clomazone e imazethapyr promove melhor controle de Chenopodiumalbum, Panicum dichotomiflorum e Ambrosia artemisiifolia. Monquero et al. (2001) constataram que a adição de chlorimuron-ethyl ao glyphosate promoveu efeito sinergístico no controle de Amaranthus hybridus e Richardia brasiliensis.

Os herbicidas chlorimuron-ethyl e imazethapyr apresentam ação em aplicações em pós-emergência, porém possuem, também, atividade residual no solo, o que pode ser benéfico quando se pretende fazer apenas uma aplicação em pós-emergência na cultura da soja $R R^{\circledR}$. Isso porque o glyphosate não apresenta ação no solo, e a realização de apenas uma aplicação em pós-emergência pode causar, dependendo do tempo de fechamento do dossel, reinfestação de plantas daninhas na área, ocasionando problemas na colheita mecanizada. Carvalho et al. (2002) observaram que a reinfestação de Euphorbia heterophylla, em área cultivada com soja, foi reduzida em $34 \%$ quando se adicionaram $10 \mathrm{~g} \mathrm{ha}^{-1}$ de chlorimuron-ethyl ao glyphosate. Roman (2002) constatou controle de $95 \%$ de Bidens pilosa 45 dias após a aplicação da mistura de glyphosate $\left(720 \mathrm{~g} \mathrm{ha}^{-1}\right)$ com chlorimuron-ethyl (10 $\left.\mathrm{g} \mathrm{ha}^{-1}\right)$ em présemeadura da cultura da soja. Valente \& Cavazzana (2000) classificaram como excelente o controle da sementeira de Bidens pilosa com a aplicação em pré-semeadura de glyphosate mais chlorimuron-ethyl.

Nesse cenário, objetivou-se com este trabalho avaliar a eficácia e a seletividade da associação dos herbicidas chlorimuron-ethyl e imazethapyr ao glyphosate no controle de plantas daninhas na cultura da soja Roundup Ready $^{\mathbb{R}}$.

\section{MATERIAL E MÉTODOS}

O experimento foi realizado no Centro Tecnológico da COMIGO, localizado no município de Rio Verde-GO, a 698 metros de altitude, com latitude de $17^{\circ} 47^{\prime} 24^{\prime \prime} \mathrm{S}$ e longitude de 
5056’31" W. O solo da área experimental é classificado como Latossolo Vermelho distrófico.

Dez dias antes da semeadura da soja Roundup Ready ${ }^{\circledR}$ (cultivar Monsoy 7878), realizada em 5.11.2005 no sistema de plantio direto, a área foi dessecada utilizando-se os herbicidas glyphosate $\left[1.440 \mathrm{~g} \mathrm{ha}^{-1}\right.$ de equivalente ácido (e.a.)] e 2,4-D (201 $\mathrm{g} \mathrm{ha}^{-1}$ e.a.).

A adubação de plantio constou da aplicação de $400 \mathrm{~kg} \mathrm{ha}^{-1}$ da fórmula 2-20-18. As sementes de soja foram previamente tratadas $(24$ horas antes do plantio) com os produtos carbendazin (30 g $100 \mathrm{~kg}^{-1}$ de sementes) e tiram (70 g $100 \mathrm{~kg}^{-1}$ de sementes). A semeadura foi realizada mecanicamente, com distribuição de 18 sementes por metro. O espaçamento utilizado entre linhas foi de $0,50 \mathrm{~m}$.

Os tratamentos herbicidas avaliados estão descritos na Tabela 1. O delineamento experimental foi o de blocos ao acaso, sendo realizadas quatro repetições. As dimensões da parcela experimental foram de $8 \mathrm{~m}$ de comprimento por $2 \mathrm{~m}$ de largura (quatro linhas de soja), totalizando $16 \mathrm{~m}^{2}$, sendo a área útil utilizada nas avaliações de $6 \mathrm{~m}^{2}$.

Aos 28 dias após a emergência da soja foi realizada a aplicação dos tratamentos herbicidas, utilizando-se um pulverizador costal com pressurização por $\mathrm{CO}_{2}$, equipado com quatro pontas de pulverização do tipo XR110-02, sendo aplicado volume de calda equivalente a $200 \mathrm{~L} \mathrm{ha}^{-1}$.

Por ocasião da aplicação dos produtos avaliados, as principais espécies infestantes presentes na área experimental, assim como as suas densidades e estádios de desenvolvimento, eram observadas na Tabela 2.

Foram realizadas duas avaliações [13 e 25 dias após a aplicação dos herbicidas (DAA)] dos sintomas de intoxicação visual nas plantas de soja provocados pelos tratamentos herbicidas, de forma visual, utilizando-se escala percentual de 0 a 100 , em que 0 representa ausência de sintomas e 100, morte das plantas. Concomitantemente, avaliou-se o controle das espécies de plantas daninhas descritas na Tabela 2. Aos 41 DAA foi avaliada a altura das plantas de soja e coletada a parte aérea de quatro plantas ao acaso por parcela, sendo colocadas em estufa de circulação forçada de ar, à temperatura constante de $65^{\circ}$, por $72 \mathrm{~h}$. Após esse período, foi realizada a pesagem para determinação da massa seca da parte aérea das plantas. Ao final do ciclo da cultura avaliou-se a produtividade, corrigindose a umidade dos grãos para $13 \%$.

Após a coleta e tabulação dos dados, procedeu-se à análise de variância, sendo as médias das variáveis significativas comparadas pelo teste de Scott-Knott a 5\%.

\section{RESULTADOS E DISCUSSÃO}

Não foram observados sintomas de intoxicação pelas plantas de soja $\mathrm{RR}^{\circledR}$ provocados pela aplicação isolada de glyphosate, independentemente da dose testada (Tabela 3). Esses dados não corroboram os encontrados por Foloni et al. (2005), que observaram leve efeito de intoxicação em plantas de soja $\mathrm{RR}^{\circledR}$ (cultivar Mons oy 8888) após aplicações isoladas de glyphosate. Na avaliação de intoxicação visual em plantas de soja $R^{\circledR}$, realizada aos 13 dias após a aplicação (DAA) dos herbicidas, constatou-se que a adição de 100,0 $\mathrm{g} \mathrm{ha}^{-1}$ de imazethapyr ao glyphosate, em qualquer dose testada, e a combinação de $10,0 \mathrm{~g} \mathrm{ha}^{-1}$ de chlorimuron-ethyl com $1.440 \mathrm{~g} \mathrm{ha}^{-1} \mathrm{de}$ glyphosate promoveram os maiores niveis de injúrias às plantas de soja $\mathrm{RR}^{\circledR}$, atingindo niveis superiores a 30\% (Tabela 3). Vidrine et al. (2002) encontraram valores para intoxicação visual em plantas de soja $R^{\circledR}{ }^{\circledR}$, provocados pela aplicação de misturas de chlorimuronethyl com glyphosate, variando de $6 \%$ a pouco mais de $30 \%$. Ellis \& Griffin (2003) observaram $13 \%$ de intoxicação em plantas de soja $R^{\circledR}$ após a aplicação de chlorimuron-ethyl $\left(6,7 \mathrm{~g} \mathrm{ha}^{-1}\right)$ com glyphosate (1.120 $\left.\mathrm{g} \mathrm{ha}^{-1}\right)$.

Diminuição da intensidade dos sintomas ocasionados pela aplicação dos herbicidas às plantas de soja $R^{\circledR}$ foi verificada aos 25 DAA em todos os tratamentos, com exceção da aplicação de 50,0 $\mathrm{g}^{\text {ha }}{ }^{-1}$ de imazethapyr em mistura com 960 ou $1.440 \mathrm{~g} \mathrm{ha}^{-1}$ de glyphosate (Tabela 3). Mesmo com a redução no nível de injúrias, a adição de 100,0 $\mathrm{g} \mathrm{ha}^{-1}$ de imazethapyr ao glyphosate nas doses de 960 ou $1.440 \mathrm{~g} \mathrm{ha}^{-1}$ acarretou os maiores niveis de intoxicação às plantas de soja $\mathrm{RR}^{\circledR}$, sendo estes, contudo, inferiores a $25 \%$. Constata-se que a adição de 
Tabela 1 - Tratamentos herbicidas utilizados no experimento. Rio Verde-GO, 2005/2006

\begin{tabular}{|c|c|c|c|}
\hline \multirow{2}{*}{ Nome comum } & \multirow{2}{*}{ Nome comercial } & Dose & Dose \\
\hline & & gi.a..$^{\prime \prime}$ ou e.a..$^{2 \prime} \mathrm{ha}^{-1}$ & g ou L p.c. ${ }^{3 /}$ ha $^{-1}$ \\
\hline 1. testemunha infestada & Testemunha infestada & - & - \\
\hline 2. testemunha capinada & Testemunha capinada & - & - \\
\hline 3. glyphosate & Round up Transorb ${ }^{\circledR}$ & 480 & 1,0 \\
\hline 4. glyphosate & Round up Transorb ${ }^{\circledR}$ & 960 & 2,0 \\
\hline 5. glyphosate & Round up Transorb ${ }^{\circledR}$ & 1.440 & 3,0 \\
\hline 6. glyphosate + chlorimuron-ethyl & Round up Transorb ${ }^{\circledR}+$ Classic $^{\circledR}$ & $480+2,5$ & $1,0+10$ \\
\hline 7. glyphosate + chlorimuron-ethyl & Round up Transorb ${ }^{\circledR}+$ Classic $^{\circledR}$ & $480+5,0$ & $1,0+20$ \\
\hline 8. glyphosate + chlorimuron-ethyl & Round up Transorb ${ }^{\circledR}+$ Classic $^{\circledR}$ & $480+10,0$ & $1,0+40$ \\
\hline 9. glyphosate + chlorimuron-ethyl & Round up Transorb $^{\circledR}+$ Classic $^{\circledR}$ & $960+2,5$ & $2,0+10$ \\
\hline 10. glyphosate + chlorimuron-ethyl & Round up Transorb $^{\circledR}+$ Classic $^{\circledR}$ & $960+5,0$ & $2,0+20$ \\
\hline 11. glyphosate + chlorimuron-ethyl & Round up Transorb $^{\circledR}+$ Classic $^{\circledR}$ & $960+10,0$ & $2,0+40$ \\
\hline 12. glyphosate + chlorimuron-ethyl & Round up Transorb $^{\circledR}+$ Classic $^{\circledR}$ & $1.440+2,5$ & $3,0+10$ \\
\hline 13. glyphosate + chlorimuron-ethyl & Round up Transorb ${ }^{\circledR}+$ Classic $^{\circledR}$ & $1.440+5,0$ & $3,0+20$ \\
\hline 14. glyphosate + chlorimuron-ethyl & Round up Transorb $^{\circledR}+$ Classic $^{\circledR}$ & $1.440+10,0$ & $3,0+40$ \\
\hline 15. glyphosate + imazethapyr & Round up Transorb $^{\circledR}+$ Pivot $^{\circledR}$ & $480+50$ & $1,0+0,5$ \\
\hline 16. glyphosate + imazethapyr & Round up Transorb ${ }^{\circledR}+$ Pivot $^{\circledR}$ & $480+100$ & $1,0+1,0$ \\
\hline 17. glyphosate + imazethapyr & Round up Transorb ${ }^{\circledR}+$ Pivot $^{\circledR}$ & $960+50$ & $2,0+0,5$ \\
\hline 18. glyphosate + imazethapyr & Round up Transorb ${ }^{\circledR}+$ Pivot $^{\circledR}$ & $960+100$ & $2,0+1,0$ \\
\hline 19. glyphosate + imazethapyr & Round up Transorb ${ }^{\circledR}+$ Pivot $^{\circledR}$ & $1.440+50$ & $3,0+0,5$ \\
\hline 20. glyphosate + imazethapyr & Round up Transorb $^{\circledR}+$ Pivot $^{\circledR}$ & $1.440+100$ & $3,0+1,0$ \\
\hline
\end{tabular}

${ }^{1 /}$ Ingrediente ativo. ${ }^{2 /}$ equivalente ácido, utilizado para o herbicida glyphosate. ${ }^{3 /}$ produto comercial.

Tabela 2 - Plantas infestantes, densidade e estádio de desenvolvimento das plantas no momento da aplicação dos herbicidas. Rio Verde-GO. 2005/2006

\begin{tabular}{|c|c|c|c|}
\hline \multicolumn{2}{|c|}{ Planta infestante } & \multirow{2}{*}{$\begin{array}{l}\text { Densidade média } \\
\left(\text { plantas } \mathrm{m}^{2}\right)\end{array}$} & \multirow{2}{*}{ Número de folhas } \\
\hline Nome científico & Nome comum & & \\
\hline Euphorbia heterophylla & leiteiro & 2 & $4-8$ \\
\hline Commelina benghalensis & trapoeraba & 3 & $2-6$ \\
\hline Chamaesyce hirta & erva-de-santa-luzia & 5 & $4-8$ \\
\hline Leucas martinicensis & mentinha & 4 & $2-6$ \\
\hline Ipomoea grandifolia & corda-de-viola & 2 & $2-6$ \\
\hline
\end{tabular}

imazethapyr ao glyphosate para aplicações em soja $\mathrm{RR}^{\circledR}$ apresenta maior risco de promover injúrias, em comparação à adição do chlorimuron-ethyl.

Todos os tratamentos que continham o herbicida imazethapyr promoveram redução na altura e na massa seca da parte aérea das plantas de soja $\mathrm{RR}^{\circledR}$ (Tabela 3). Krausz \& Young (2001) constataram que a aplicação do herbicida imazethapyr atrasou o ciclo da cultura da soja
$\mathrm{RR}^{\circledR}$. Todos os tratamentos que possuíam o herbicida chlorimuron-ethyl não provocaram diminuições na altura e na massa seca da parte aérea das plantas de soja $R^{\circledR}$. Como observado nas avaliações dos sintomas de intoxicação, a adição de imazethapyr ao glyphosate para aplicações em pós-emergência em soja $R R^{\circledR}$ mostrou maiores danos sobre o crescimento e o acúmulo de massa seca, em comparação à adição do chlorimuron-ethyl. 
Tabela 3 - Intoxicação visual, altura de plantas e massa seca da parte aérea de plantas de soja RR ${ }^{\circledR}$ (cultivar Monsoy 7878), após a aplicação de diferentes tratamentos herbicidas em pós-emergência. Rio Verde-GO, 2005/2006

\begin{tabular}{|l|c|c|c|c|c|}
\hline \multirow{2}{*}{ Tratamento } & \multirow{2}{*}{ Dose } & \multicolumn{2}{|c|}{ Intoxicação (\%) } & $\begin{array}{c}\text { Altura de } \\
\text { plantas (cm) }\end{array}$ & $\begin{array}{c}\text { Massa seca da } \\
\text { parte aérea (g) }\end{array}$ \\
\cline { 2 - 6 } & $\begin{array}{c}\text { g i.a. }{ }^{\prime \prime} \text { ou } \\
\text { e.a. }{ }^{\prime} \text { ha }^{-1}\end{array}$ & 13 DAA $^{3 /}$ & 25 DAA & 41 DAA & 41 DAA \\
\hline 1. testemunha infestada & - & $0 \mathrm{e}$ & $0 \mathrm{e}$ & $76,9 \mathrm{a}$ & $15,38 \mathrm{a}$ \\
\hline 2. testemunha capinada & - & $0 \mathrm{e}$ & $0 \mathrm{e}$ & $75,8 \mathrm{a}$ & $15,16 \mathrm{a}$ \\
\hline 3. glyphosate & 480 & $0 \mathrm{e}$ & $0 \mathrm{e}$ & $75,0 \mathrm{a}$ & $15,00 \mathrm{a}$ \\
\hline 4. glyphosate & 960 & $0 \mathrm{e}$ & $0 \mathrm{e}$ & $76,7 \mathrm{a}$ & $15,34 \mathrm{a}$ \\
\hline 5. glyphosate & 1.440 & $0 \mathrm{e}$ & $0 \mathrm{e}$ & $75,8 \mathrm{a}$ & $15,16 \mathrm{a}$ \\
\hline 6. glyphosate + chlorimuron-ethyl & $480+2,5$ & $4 \mathrm{~d}$ & $0 \mathrm{e}$ & $77,3 \mathrm{a}$ & $15,46 \mathrm{a}$ \\
\hline 7.glyphosate + chlorimuron-ethyl & $480+5,0$ & $1 \mathrm{e}$ & $0 \mathrm{e}$ & $78,7 \mathrm{a}$ & $15,73 \mathrm{a}$ \\
\hline 8. glyphosate + chlorimuron-ethyl & $480+10,0$ & $15 \mathrm{c}$ & $5 \mathrm{~d}$ & $76,9 \mathrm{a}$ & $15,38 \mathrm{a}$ \\
\hline 9. glyphosate + chlorimuron-ethyl & $960+2,5$ & $1 \mathrm{e}$ & $0 \mathrm{e}$ & $75,8 \mathrm{a}$ & $15,17 \mathrm{a}$ \\
\hline 10. glyphosate + chlorimuron-ethyl & $960+5,0$ & $13 \mathrm{c}$ & $7 \mathrm{~d}$ & $76,0 \mathrm{a}$ & $15,21 \mathrm{a}$ \\
\hline 11. glyphosate + chlorimuron-ethyl & $960+10,0$ & $25 \mathrm{~b}$ & $15 \mathrm{~b}$ & $74,0 \mathrm{a}$ & $14,81 \mathrm{a}$ \\
\hline 12. glyphosate + chlorimuron-ethyl & $1.440+2,5$ & $3 \mathrm{~d}$ & $0 \mathrm{e}$ & $77,1 \mathrm{a}$ & $15,43 \mathrm{a}$ \\
\hline 13. glyphosate + chlorimuron-ethyl & $1.440+5,0$ & $23 \mathrm{~b}$ & $9 \mathrm{c}$ & $74,3 \mathrm{a}$ & $14,86 \mathrm{a}$ \\
\hline 14. glyphosate + chlorimuron-ethyl & $1.440+10,0$ & $33 \mathrm{a}$ & $10 \mathrm{c}$ & $74,3 \mathrm{a}$ & $14,86 \mathrm{a}$ \\
\hline 15. glyphosate + imazethapyr & $480+50$ & $3 \mathrm{~d}$ & $0 \mathrm{e}$ & $71,9 \mathrm{~b}$ & $14,38 \mathrm{~b}$ \\
\hline 16. glyphosate + imazethapyr & $480+100$ & $33 \mathrm{a}$ & $15 \mathrm{~b}$ & $69,8 \mathrm{~b}$ & $13,97 \mathrm{~b}$ \\
\hline 17. glyphosate + imazethapyr & $960+50$ & $13 \mathrm{c}$ & $13 \mathrm{~b}$ & $71,8 \mathrm{~b}$ & $14,35 \mathrm{~b}$ \\
\hline 18. glyphosate + imazethapyr & $960+100$ & $33 \mathrm{a}$ & $20 \mathrm{a}$ & $71,1 \mathrm{~b}$ & $14,22 \mathrm{~b}$ \\
\hline 19. glyphosate + imazethapyr & $1.440+50$ & $10 \mathrm{c}$ & $13 \mathrm{~b}$ & $72,9 \mathrm{~b}$ & $14,58 \mathrm{~b}$ \\
\hline 20. glyphosate + imazethapyr & $1.440+100$ & $33 \mathrm{a}$ & $23 \mathrm{a}$ & $71,2 \mathrm{~b}$ & $14,23 \mathrm{~b}$ \\
\hline
\end{tabular}

${ }^{1 /}$ Ingrediente ativo. ${ }^{2 /}$ equivalente ácido, utilizado para o herbicida glyphosate. ${ }^{3 /}$ dias após a aplicação.

Médias seguidas de mesma letra não diferem pelo critério de Scott Knott a 5\%.

O controle de Euphorbia heterophylla respondeu positivamente ao incremento das doses do glyphosate, seja aplicado isoladamente ou em mistura com chlorimuron-ethyl ou imazethapyr. No entanto, a utilização do glyphosate isolado não proporcionou controle satisfatório dessa invasora aos $25 \mathrm{DAA}$, mesmo na maior dose avaliada (1.440 $\mathrm{g} \mathrm{ha}^{-1}$ ) (Tabela 4). Aos 13 DAA, a aplicação dos tratamentos glyphosate $\left(960 \mathrm{~g} \mathrm{ha}^{-1}\right)$ mais imazethapyr $\left(100,0 \mathrm{~g} \mathrm{ha}^{-1}\right)$ e glyphosate $\left(1.440 \mathrm{~g} \mathrm{ha}^{-1}\right)$ mais imazethapyr (50,0 ou 100,0 $\mathrm{g} \mathrm{ha}^{-1}$ ) resultou nos maiores niveis de controle. Todavia, na segunda avaliação (25 DAA), maiores niveis de controle de E. heterophylla foram verificados com a utilização das combinações de $1.440 \mathrm{~g} \mathrm{ha}^{-1}$ de glyphosate com 50,0 ou 100,0 $\mathrm{g} \mathrm{ha}^{-1}$ de imazethapyr e $1.440 \mathrm{~g} \mathrm{ha}^{-1}$ de glyphosate com
5,0 ou 10,0 $\mathrm{g} \mathrm{ha}^{-1}$ de chlorimuron-ethyl, não diferindo da testemunha capinada.

A aplicação isolada de glyphosate nas doses de 480, 960 e $1.440 \mathrm{~g} \mathrm{ha}^{-1}$ não resultou em controle satisfatório da planta daninha Commelina benghalensis, sendo verificado aos 25 DAA controle máximo de $55 \%$ (Tabela 4). As combinações de glyphosate a 960 e $1.440 \mathrm{~g} \mathrm{ha}^{-1} \mathrm{com}$ imazethapyr a 50,0 e 100,0 $\mathrm{g} \mathrm{ha}^{-1}$ e as misturas de $960 \mathrm{~g} \mathrm{ha}^{-1}$ de glyphosate com 10,0 $\mathrm{g} \mathrm{ha}^{-1}$ de chlorimuron-ethyl e de $1.440 \mathrm{~g} \mathrm{ha}^{-1}$ de glyphosate com 5,0 ou $10,0 \mathrm{~g} \mathrm{ha}^{-1}$ de chlorimuron-ethyl apresentaram aos 13 DAA os melhores niveis de controle dessa espécie. No entanto, aos 25 DAA, apenas as misturas de $1.440 \mathrm{~g} \mathrm{ha}^{-1}$ de glyphosate com 50,0 ou $100,0 \mathrm{~g} \mathrm{ha}^{-1}$ de imazethapyr ou com 10,0 $\mathrm{g} \mathrm{ha}^{-1}$ 
Tabela 4 - Controle de leiteiro (Euphorbia heterophylla), trapoeraba (Commelina benghalensis) e erva-de-santa luzia (Chamaesyce hirta) por diferentes tratamentos herbicidas aplicados em pós-emergência. Rio Verde-GO, 2005/2006

\begin{tabular}{|c|c|c|c|c|c|c|c|}
\hline \multirow{3}{*}{ Tratamento } & \multirow{3}{*}{$\begin{array}{c}\text { Dose } \\
\text { g i.a. }{ }^{\frac{1}{}} \text { ou } \\
\text { e.a. } .^{\prime \prime} \mathrm{ha}^{-1}\end{array}$} & \multicolumn{6}{|c|}{ Controle $(\%)$} \\
\hline & & \multicolumn{2}{|c|}{ E. heterophylla } & \multicolumn{2}{|c|}{ C. benghalensis } & \multicolumn{2}{|c|}{ C. hirta } \\
\hline & & $13 \mathrm{DAA}^{3 /}$ & 25 DAA & 13 DAA & 25 DAA & 13 DAA & 25 DAA \\
\hline 1. testemunha infestada & - & $0 \mathrm{~g}$ & $0 \mathrm{i}$ & $0 \mathrm{f}$ & $0 \mathrm{j}$ & $0 \mathrm{e}$ & $0 \mathrm{k}$ \\
\hline 2. testemunha capinada & - & $100 \mathrm{a}$ & $100 \mathrm{a}$ & $100 \mathrm{a}$ & $100 \mathrm{a}$ & $100 \mathrm{a}$ & $100 \mathrm{a}$ \\
\hline 3. glyphosate & 480 & $43 \mathrm{e}$ & $40 \mathrm{~g}$ & $23 \mathrm{e}$ & $30 \mathrm{i}$ & $30 \mathrm{~d}$ & $30 \mathrm{j}$ \\
\hline 4. glyphosate & 960 & $65 d$ & $55 \mathrm{f}$ & $53 \mathrm{~d}$ & $40 \mathrm{~g}$ & $78 \mathrm{~b}$ & $55 \mathrm{f}$ \\
\hline 5. glyphosate & 1.440 & $83 \mathrm{~b}$ & $65 \mathrm{e}$ & $68 c$ & $55 \mathrm{e}$ & $83 \mathrm{~b}$ & $65 d$ \\
\hline 6. glyphosate + chlorimuron-ethyl & $480+2,5$ & $31 \mathrm{f}$ & $30 \mathrm{~h}$ & $43 \mathrm{~d}$ & $35 \mathrm{~h}$ & $25 \mathrm{~d}$ & $30 \mathrm{j}$ \\
\hline 7. glyphosate + chlorimuron-ethyl & $480+5,0$ & $40 \mathrm{e}$ & $40 \mathrm{~g}$ & $53 \mathrm{~d}$ & $40 \mathrm{~g}$ & $30 \mathrm{~d}$ & $35 \mathrm{i}$ \\
\hline 8. glyphosate + chlorimuron-ethyl & $480+10,0$ & $45 \mathrm{e}$ & $40 \mathrm{~g}$ & $60 \mathrm{~d}$ & $50 \mathrm{f}$ & $25 \mathrm{~d}$ & $40 \mathrm{~h}$ \\
\hline 9. glyphosate + chlorimuron-ethyl & $960+2,5$ & $60 \mathrm{~d}$ & $65 \mathrm{e}$ & $65 \mathrm{c}$ & $70 \mathrm{~d}$ & $78 \mathrm{~b}$ & $60 \mathrm{e}$ \\
\hline 10. glyphosate + chlorimuron-ethyl & $960+5,0$ & $75 \mathrm{c}$ & $73 d$ & $70 \mathrm{c}$ & $75 d$ & $83 \mathrm{~b}$ & $60 \mathrm{e}$ \\
\hline 11. glyphosate + chlorimuron-ethyl & $960+10,0$ & $85 \mathrm{~b}$ & $80 \mathrm{c}$ & $80 \mathrm{~b}$ & $75 d$ & $90 \mathrm{a}$ & $70 \mathrm{~d}$ \\
\hline 12. glyphosate + chlorimuron-ethyl & $1.440+2,5$ & $90 \mathrm{~b}$ & $85 b$ & $65 c$ & $75 d$ & $95 \mathrm{a}$ & $80 \mathrm{c}$ \\
\hline 13. glyphosate + chlorimuron-ethyl & $1.440+5,0$ & $90 \mathrm{~b}$ & $98 \mathrm{a}$ & $83 \mathrm{~b}$ & $80 \mathrm{c}$ & $95 \mathrm{a}$ & $90 \mathrm{~b}$ \\
\hline 14. glyphosate + chlorimuron-ethyl & $1.440+10,0$ & $90 \mathrm{~b}$ & $100 \mathrm{a}$ & $78 \mathrm{~b}$ & $88 \mathrm{~b}$ & $95 \mathrm{a}$ & $95 \mathrm{a}$ \\
\hline 15. glyphosate + imazethapyr & $480+50$ & $85 \mathrm{~b}$ & $55 \mathrm{f}$ & $50 \mathrm{~d}$ & $50 \mathrm{f}$ & $60 c$ & $50 \mathrm{~g}$ \\
\hline 16. glyphosate + imazethapyr & $480+100$ & $90 \mathrm{~b}$ & $80 \mathrm{c}$ & $73 \mathrm{c}$ & $55 \mathrm{e}$ & $65 \mathrm{c}$ & $55 \mathrm{f}$ \\
\hline 17. glyphosate + imazethapyr & $960+50$ & $90 \mathrm{~b}$ & $70 \mathrm{~d}$ & $76 \mathrm{~b}$ & $60 \mathrm{e}$ & $78 \mathrm{~b}$ & $55 \mathrm{f}$ \\
\hline 18. glyphosate + imazethapyr & $960+100$ & $95 \mathrm{a}$ & $80 \mathrm{c}$ & $83 \mathrm{~b}$ & $80 \mathrm{c}$ & $80 \mathrm{~b}$ & $60 \mathrm{e}$ \\
\hline 19. glyphosate + imazethapyr & $1.440+50$ & $95 \mathrm{a}$ & $100 \mathrm{a}$ & $78 \mathrm{~b}$ & $85 \mathrm{~b}$ & $95 \mathrm{a}$ & $90 \mathrm{~b}$ \\
\hline 20. glyphosate + imazethapyr & $1.440+100$ & $95 \mathrm{a}$ & $100 \mathrm{a}$ & $83 \mathrm{~b}$ & $83 \mathrm{~b}$ & $93 \mathrm{a}$ & $93 \mathrm{~b}$ \\
\hline
\end{tabular}

$\underline{1}$ Ingrediente ativo. ${ }^{2 /}$ equivalente ácido, utilizado para o herbicida glyphosate. ${ }^{3 /}$ dias após a aplicação.

Médias seguidas de mesma letra não diferem pelo critério de Scott Knott a 5\%.

de cholrimuron-ethyl permaneceram como os melhores tratamentos químicos para controle de $C$. benghalensis, porém não se igualando à testemunha capinada (Tabela 4). Apesar de a adição dos herbicidas imazethapyr e chlorimuron-ethyl promover melhora acentuada em relação à aplicação isolada do glyphosate, nenhuma associação desses herbicidas apresentou controle superior a $90 \%$ de C. benghalensis - fato preocupante, pois essa espécie invasora vem se disseminando em taxas extremamente elevadas nas lavouras do cerrado brasileiro.

O aumento das doses de glyphosate, quando aplicado sem associação com outros herbicidas, resultou em incremento no controle de Chamaesyce hirta, contudo não atingindo niveis satisfatórios. $\mathrm{Na}$ avaliação do controle de $C$. hirta, realizada aos 13 DAA, constatou-se que as misturas de $960 \mathrm{~g} \mathrm{ha}^{-1}$ de glyphosate com
$10,0 \mathrm{~g} \mathrm{ha}^{-1}$ de chlorimuron-ethyl, $1.440 \mathrm{~g} \mathrm{ha}^{-1}$ de glyphosate com 2,5, 5,0 e 10,0 $\mathrm{g} \mathrm{ha}^{-1}$ de chlorimuron-ethyl e $1.440 \mathrm{~g}^{\text {ha }}{ }^{-1}$ de glyphosate com 50,0 e $100,0 \mathrm{~g} \mathrm{ha}^{-1}$ de imazethapyr foram as mais eficientes em relação aos demais tratamentos (Tabela 4). Todavia, aos 25 DAA, apenas $1.440 \mathrm{~g} \mathrm{ha}^{-1}$ de glyphosate com 10,0 $\mathrm{g} \mathrm{ha}^{-1}$ de chlorimuron-ethyl foi o tratamento químico que não diferiu da testemunha mantida no limpo, apresentando controle de C. hirta de 95\%. Esses resultados indicam que a adição de chlorimuron-ethyl ao glyphosate é extremamente benéfica para controle de C. hirta em pós-emergência em lavouras de soja $R^{\circledR}$.

A espécie daninha Leucas martinicensis também não foi controlada eficientemente pela ação isolada de glyphosate em qualquer das doses testadas, e a aplicação da dose de $1.440 \mathrm{~g} \mathrm{ha}^{-1}$ foi a que proporcionou o melhor 
controle (65\%) aos 25 DAA (Tabela 5). Os tratamentos quimicos que promoveram os melhores niveis de controle aos 13 e aos 25 DAA foram $1.440 \mathrm{~g} \mathrm{ha}^{-1}$ de glyphosate em associação com 50,0 ou $100,0 \mathrm{~g} \mathrm{ha}^{-1}$ de imazethapyr, equiparando-se ao controle observado na testemunha capinada. Esses resultados mostram que o imazethapyr se apresenta como excelente herbicida para ser usado em áreas cultivadas com soja $\mathrm{RR}^{\circledR}$ infestadas com L. martinicensis. Também, ressalta-se que a adição de todas as doses de chlorimuron-ethyl ao glyphosate aplicado na dose de $1.440 \mathrm{~g}^{\text {ha }}{ }^{-1}$ proporcionou controle de $L$. martinicensis, aos 25 DAA, acima de $90 \%$, porém inferior ao controle observado com imazethapyr em mistura com a mesma dose de glyphosate.

Como as demais espécies daninhas avaliadas, Ipomoea grandifolia apresentou tolerância à aplicação isolada do glyphosate em todas as doses, sendo de $55 \%$ o melhor controle, observado aos 25 DAA com a utilização desse herbicida (Tabela 5). A mistura de $1.440 \mathrm{~g} \mathrm{ha}^{-1}$ de glyphosate com 100,0 $\mathrm{g} \mathrm{ha}^{-1}$ de imazethapyr foi a que melhor controlou I. grandifolia aos 13 DAA, com controle médio de $80 \%$. Contudo, na segunda avaliação (25 DAA) as combinações de $1.440 \mathrm{~g} \mathrm{ha}^{-1}$ de glyphosate com 5,0 ou $10,0 \mathrm{~g} \mathrm{ha}^{-1}$ de chlorimuron-ethyl promoveram controle médio de $85 \%$, mostrando-se como as mais eficazes no controle dessa planta daninha. Esses resultados estão de acordo com os encontrados por Norsworthy \& Grey (2004), os quais verificaram que a associação de chlorimuron-ethyl ao glyphosate incrementou o controle de Ipomoea hederacea e Ipomoea purpurea. Nenhum dos tratamentos químicos testados apresentou controle de I. grandifolia estatisticamente semelhante ao da testemunha capinada, ou mesmo superior a $90 \%$, o que demonstra que outras opções de herbicidas para aplicação conjunta com glyphosate devem ser avaliadas, visando o controle dessa invasora.

Não foram detectadas diferenças entre os tratamentos quanto à produtividade de grãos (Tabela 6), demonstrando que a interferência causada pela presença das plantas daninhas

Tabela 5 - Controle de mentinha (Leucas martinicensis) e corda-de-viola (Ipomoea grandifolia) por diferentes tratamentos herbicidas aplicados em pós -emergência. Rio Verde-GO, 2005/2006

\begin{tabular}{|c|c|c|c|c|c|}
\hline \multirow{3}{*}{ Tratamento } & \multirow{3}{*}{$\begin{array}{c}\text { Dose } \\
\text { g i.a. } .^{1 /} \text { ou } \\
\text { e.a. } .^{2 !} \mathrm{ha}^{-1}\end{array}$} & \multicolumn{4}{|c|}{ Controle (\%) } \\
\hline & & \multicolumn{2}{|c|}{ L. martinicensis } & \multicolumn{2}{|c|}{ I. grandifolia } \\
\hline & & $13 \mathrm{DAA}^{3 /}$ & $25 \mathrm{DAA}$ & 13 DAA & 25 DAA \\
\hline 1. testemunha infestada & - & $0 \mathrm{j}$ & $0 \mathrm{j}$ & $0 \mathrm{~h}$ & $0 \mathrm{j}$ \\
\hline 2. testemunha capinada & - & $100 \mathrm{a}$ & $100 \mathrm{a}$ & $100 \mathrm{a}$ & $100 \mathrm{a}$ \\
\hline 3. glyphosate & 480 & $34 \mathrm{i}$ & $40 \mathrm{~h}$ & $48 \mathrm{~g}$ & $50 \mathrm{~g}$ \\
\hline 4. glyphosate & (9) & $55 \mathrm{~g}$ & $40 \mathrm{~h}$ & $55 \mathrm{f}$ & $50 \mathrm{~g}$ \\
\hline 5. glyphosate & 1.440 & $73 \mathrm{e}$ & $65 \mathrm{e}$ & $65 \mathrm{~d}$ & $55 \mathrm{f}$ \\
\hline 6. glyphosate + chlorimuron -ethyl & $480+2,5$ & $33 \mathrm{i}$ & $25 \mathrm{i}$ & $40 \mathrm{~g}$ & $40 \mathrm{i}$ \\
\hline 7. glyphosate + chlorimuron -ethyl & $480+5,0$ & $53 \mathrm{~g}$ & $50 \mathrm{~g}$ & $50 \mathrm{f}$ & $45 \mathrm{~h}$ \\
\hline 8. glyphosate + chlorimuron-ethyl & $480+10,0$ & $65 \mathrm{f}$ & $50 \mathrm{~g}$ & $50 \mathrm{f}$ & $50 \mathrm{~g}$ \\
\hline 9. glyphosate + chlorimuron-ethyl & $960+2,5$ & $78 \mathrm{~d}$ & $75 \mathrm{~d}$ & $45 \mathrm{~g}$ & $60 \mathrm{e}$ \\
\hline 10. glyphosate + chlorimuron-ethyl & $960+5,0$ & $85 \mathrm{c}$ & $80 \mathrm{c}$ & $60 \mathrm{e}$ & $65 \mathrm{e}$ \\
\hline 11. glyphosate + chlorimuron-ethyl & $960+10,0$ & $90 \mathrm{~b}$ & $81 \mathrm{c}$ & $65 \mathrm{~d}$ & $75 c$ \\
\hline 12. glyphosate + chlorimuron-ethyl & $1.440+2,5$ & $90 \mathrm{~b}$ & $93 \mathrm{~b}$ & $50 \mathrm{f}$ & $80 \mathrm{c}$ \\
\hline 13. glyphosate + chlorimuron-ethyl & $1.440+5,0$ & $90 \mathrm{~b}$ & $93 \mathrm{~b}$ & $60 \mathrm{e}$ & $85 \mathrm{~b}$ \\
\hline 14. glyphosate + chlorimuron-ethyl & $1.440+10,0$ & $90 \mathrm{~b}$ & $95 \mathrm{~b}$ & $73 c$ & $85 \mathrm{~b}$ \\
\hline 15. glyphosate + imazethapyr & $480+50$ & $40 \mathrm{~h}$ & $50 \mathrm{~g}$ & $43 \mathrm{~g}$ & $50 \mathrm{~g}$ \\
\hline 16. glyphosate + imazethapyr & $480+100$ & $64 \mathrm{f}$ & $55 \mathrm{f}$ & $43 \mathrm{~g}$ & $55 \mathrm{f}$ \\
\hline 17. glyphosate + imazethapyr & $960+50$ & $65 \mathrm{f}$ & $70 \mathrm{~d}$ & $53 \mathrm{f}$ & $55 \mathrm{f}$ \\
\hline 18. glyphosate + imazethapyr & $960+100$ & $65 \mathrm{f}$ & $83 c$ & $53 \mathrm{f}$ & $60 \mathrm{e}$ \\
\hline 19. glyphosate + imazethapyr & $1.440+50$ & $95 \mathrm{a}$ & $100 \mathrm{a}$ & $60 \mathrm{e}$ & $70 d$ \\
\hline 20. glyphosate + imazethapyr & $1.440+100$ & $95 \mathrm{a}$ & $100 \mathrm{a}$ & $80 \mathrm{~b}$ & $75 \mathrm{c}$ \\
\hline
\end{tabular}

${ }^{1 /}$ Ingrediente ativo. ${ }^{2 /}$ equivalente ácido, utilizado para o herbicida glyphosate. ${ }^{3 /}$ dias após a aplicação.

Médias seguidas de mesma letra não diferem pelo critério de Scott Knott, a 5\%. 
Tabela 6 - Produtividade da cultura da soja $\mathrm{RR}^{\circledR}$ (cultivar Monsoy 7878) após a aplicação de diferentes tratamentos herbicidas em pós-emergência. Rio Verde, GO, 2005/2006

\begin{tabular}{|l|c|c|}
\hline Tratamento & Dose $\left(\mathrm{g}\right.$ i.a. ${ }^{1 /}$ ou e.a..$\left.^{2 /} \mathrm{ha}^{-1}\right)$ & Produtividade $\left(\mathrm{kg}\right.$ ha $\left.{ }^{-1}\right)$ \\
\hline 1. testemunha infestada & - & $2.900 \mathrm{a}$ \\
\hline 2. testemunha capinada & - & $3.082 \mathrm{a}$ \\
\hline 3. glyphosate & 480 & $2.820 \mathrm{a}$ \\
\hline 4. glyphosate & 960 & $2.583 \mathrm{a}$ \\
\hline 5. glyphosate & 1.440 & $2.854 \mathrm{a}$ \\
\hline 6. glyphosate + chlorimuron-ethyl & $480+2,5$ & $2.914 \mathrm{a}$ \\
\hline 7. glyphosate + chlorimuron-ethyl & $480+5,0$ & $2.951 \mathrm{a}$ \\
\hline 8. glyphosate + chlorimuron-ethyl & $480+10,0$ & $2.877 \mathrm{a}$ \\
\hline 9. glyphosate + chlorimuron-ethyl & $960+2,5$ & $2.894 \mathrm{a}$ \\
\hline 10. glyphosate + chlorimuron -ethyl & $960+5,0$ & $2.811 \mathrm{a}$ \\
\hline 11. glyphosate + chlorimuron -ethyl & $960+10,0$ & $2.685 \mathrm{a}$ \\
\hline 12. glyphosate + chlorimuron -ethyl & $1.440+2,5$ & $2.960 \mathrm{a}$ \\
\hline 13. glyphosate + chlorimuron -ethyl & $1.440+5,0$ & $2.965 \mathrm{a}$ \\
\hline 14. glyphosate + chlorimuron -ethyl & $1.440+10,0$ & $2.845 \mathrm{a}$ \\
\hline 15. glyphosate + imazethapyr & $480+50$ & $2.588 \mathrm{a}$ \\
\hline 16. glyphosate + imazethapyr & $480+100$ & $2.614 \mathrm{a}$ \\
\hline 17. glyphosate + imazethapyr & $960+50$ & $2.637 \mathrm{a}$ \\
\hline 18. glyphosate + imazethapyr & $960+100$ & $2.703 \mathrm{a}$ \\
\hline 19. glyphosate + imazethapyr & $1.440+50$ & $2.460 \mathrm{a}$ \\
\hline 20. glyphosate + imazethapyr & $1.440+100$ & $2.697 \mathrm{a}$ \\
\hline
\end{tabular}

${ }^{\mathrm{L}}$ Ingrediente ativo. ${ }^{2 /}$ equivalente ácido, utilizado para o herbicida glyphosate.

Médias seguidas de mesma letra não diferem pelo critério de Scott Knott, a $5 \%$.

não controladas ou parcialmente controladas pelos tratamentos herbicidas não foi suficiente para afetar essa característica. Vidrine et al. (2002) verificaram que o aumento no controle das plantas daninhas promovido pela adição de chlorimuron-ethyl ao glyphosate não refletiu em aumento de produtividade da cultura da soja $R^{\circledR}$. Estudos realizados por Krausz et al. (2001) e Ellis \& Grifin (2003) constataram que a adição de latifolicidas ao glyphosate não promoveu redução de produtividade na cultura da soja $R^{\circledR}$. A baixa densidade de plantas daninhas (Tabela 2) associada a um rápido e vigoroso crescimento das plantas de soja $R^{\circledR}$ pode ser o motivo da não-ocorrência de diferenças entre os tratamentos quanto à produtividade de grãos.

\section{LITERATURA CITADA}

CARVALHO, F. T. et al. Manejo químico das plantas daninhas Euphorbia heterophylla e Bidens pilosa em sistema de plantio direto da cultura de soja. Planta Daninha, v. 20, p. 145-150, 2002.

ELLIS, J. M.; GRIFFIN, J. L. Glyphosate and broadleaf herbicide mixtures for soybean (Glycine max). Weed Technol., v. 17, p. 21-27, 2003.
FOLONI, L. L. et al. Aplicação de glifosato em pósemergência, em soja transgênica cultivada no cerrado. R. Bras. Herbic., v. 4, p. 47-58, 2005.

GAZZIERO, D. L. P. et al. Deposição de glyphosate aplicado para controle de plantas daninhas em soja transgênica. Planta Daninha, v. 24, p. 173-181, 2006.

JOHNSON, B. F. et al. Herbicide effects on visible injury, leaf area, and yield of glyphosate-resistant soybean (Glycine max). Weed Technol., v. 16, p. 554-566, 2002.

KRAUSZ, R. F. et al. Influence of weed competition and herbicides on glyphosate-resistant soybean (Glycine max). Weed Technol., v. 15, p. 530-534, 2001.

KRAUSZ, R. F.; YOUNG, B. G. Response of double-crop glyphosate-resistant soybean (Glycine max) to broadleaf herbicides. Weed Technol., v. 15, p. 300-305, 2001.

MONQUERO, P. A.; CHRISTOFFOLETI, P. J.; SANTOS, C. T. D. Glyphosate em mistura com herbicidas alternativos para o manejo de plantas daninhas. Planta Daninha, v. 19, p. 375-380, 2001.

NORSWORTHY, J. K.; GREY, T. L. Addition of nonionic surfactant to glyphosate plus chlorimuron. Weed Technol., v. 18, p. 588-593, 2004.

ROMAN, E. S. Eficácia de herbicidas na dessecação e no controle residual de plantas daninhas no sistema desseque e plante. R. Bras. Herb., v. 3, p. 45-49, 2002. 
VALENTE, T. O.; CAVAZZANA, M. A. Efeito residual de chlorimuron-ethyl aplicado em mistura com glyphosate na dessecação de plantas daninhas. R. Bras. Herb., v. 1, p. 173 $178,2000$.

VANGESSEL, M. J.; AYENI, A. A.; MAJEK, B. A. Glyphosate in full-season no-till glyphosate resistant soybean: role of preplant applications and residual herbicides. Weed Technol., v. 15, p. 714-724, 2001.
VANLIESHOUT, L. A.; LOUX, M. M. Interactions of glyphosate with residual herbicides in no-till soybean (Glycine max) production. Weed Technol., v. 14, p. 480487, 2000.

VIDRINE, R. P.; GRIFFIN, J. L.; BLOUIN, D. C.

Evaluation of reduced rates of glyphosate and chlorimuron in glyphosate-resistant soybean (Glycine max). Weed Technol., v. 16, p. 731-736, 2002. 